\title{
Charge Transport and Phase Behavior of Imidazolium-Based Ionic Liquid Crystals from Fully Atomistic Simulations
}

\author{
Michael J. Quevillon (D) and Jonathan K. Whitmer* (D) \\ Department of Chemical and Biomolecular Engineering, University of Notre Dame, Notre Dame, IN 46556, USA; \\ mquevill@nd.edu \\ * Correspondence: jwhitme1@nd.edu
}

Received: 10 November 2017; Accepted: 20 December 2017; Published: 2 January 2018

\section{Check for updates}

\begin{abstract}
Ionic liquid crystals occupy an intriguing middle ground between room-temperature ionic liquids and mesostructured liquid crystals. Here, we examine a non-polarizable, fully atomistic model of the 1-alkyl-3-methylimidazolium nitrate family using molecular dynamics in the constant pressure-constant temperature ensemble. These materials exhibit a distinct "smectic" liquid phase, characterized by layers formed by the molecules, which separate the ionic and aliphatic moieties. In particular, we discuss the implications this layering may have for electrolyte applications.
\end{abstract}

Keywords: ionic liquids; liquid crystals; ionic liquid crystals; phase; charge transport

\section{Introduction}

The intersection of the fields of ionic liquids and liquid crystals is a relatively new sector of materials science, but has garnered much research in recent years [1-4], as ionic liquid crystals begin to show promise as useful molecules in tunable conductive materials $[5,6]$ or as greener solvents $[7,8]$, for example. Liquid crystalline phases are of interest due to their innate mesostructure, arising from energetically- or entropically-driven alignment of molecules, or from phase separation arrested by molecular structure. This results in many different ordered phases [5,9-11], including multiple nematic, smectic, and columnar phases [10], among others. This wide variety of mesophases split our traditional notion of a liquid, offering variable degrees and types of orientational and translational order without crystallization. Thermotropic liquid crystals can lose their partially ordered nature at high temperatures and form a completely disordered isotropic liquid phase [10], while lyotropic liquid crystals change phases at different concentrations, allowing for surfactant-like mesophases, such as micellar or bicontinuous cubic phases [12]. In solvent-free situations, liquid crystals commonly regarded as lyotropic behave thermotropically, exhibiting multiple interesting mesophases. Typical lyotropic liquid crystals are aqueous-solvated surfactants [13,14], with polar or charged head groups and aliphatic tails; the household soap sodium dodecyl sulfate (SDS) is one such example. There, the headgroup exhibits a relatively "hard" sulfate ion associating with a compact sodium cation in the absence of a solvent. When the solution is removed, this results in a solid phase at room temperature [15]. If the headgroup is made bulkier, spreading out the charge and limiting close cation-anion contact, a liquid phase can be promoted at room temperature; this is the case in standard ionic liquids [16]. While most ionic liquids lack the extended alkyl chain similar to that found in SDS, thereby exhibiting only an isotropic liquid phase, addition of an extended tail can promote ordering via micro-phase separation, resulting in mesostructured ionic liquids, hence ionic liquid crystals. 
Mesophases have long been observed in molten salt systems [17], but tuning the molecules to reach liquid crystal phases closer to room-temperature has been of more recent interest. Molecules studied at the intersection of these two fields are often based around imidazolium or viologen groups [18], with elongated alkyl tails attached to one or more ions. The counter-ion can be either a "simple" ionic liquid species (such as $\left[\mathrm{PF}_{6}\right]^{-}$or $\left[\mathrm{BF}_{4}\right]^{-}$) or another ion with a long alkyl chain. The alkyl chain has a significant influence on the ordering properties observed, and the range of temperatures for which a given structure is stable, as it controls the relative energetic considerations for the formation of ordered ionic and apolar domains which drives ionic liquid crystal formation [19]. For a material to be considered an ionic liquid crystal with partial long-range order, the threshold of alkyl chain length [20] varies with species and with counter-ion, indicating that a balance of electrostatic and van der Waals forces strongly influences the phases. The dominant order in ionic liquid crystal phases is one of lamellar or smectic character, typified by association and alignment of the long alkyl chains, with charged moieties forming layers in the material [21]. These phases are highly reminiscent of the types of phases formed by lyotropic surfactants, with the caveat that there is no additional solvent in the ionic liquid crystal phase. Different structures and degrees of ordering in ionic liquid crystals lead to material properties that can be harnessed for various uses. Ionic liquids have very promising applications as solvents [22,23], battery electrolytes [24,25], in $\mathrm{CO}_{2}$ sequestration [26,27], and in nonlinear-optical and photonic devices [28]. These properties can be further controlled when liquid crystal ordering is incorporated. As one example, smectic ionic liquid crystals used in battery electrolytes permit directionally-dependent ionic conductivity, much as is seen in block copolymer electrolytes [29].

Though significant benefits are present, these materials are highly nontrivial to synthesize. Hence, to guide synthetic chemists in determining useful targets, accurate computer simulations are of great utility. One can adopt many levels of description in simulations depending on the specificity with which one wants to sample configurational space. One such simple model utilizing Gay-Berne [30] and Lennard-Jones molecules is able to stabilize ionic liquid crystal phases by tuning the charge on each generic particle [31], however with some loss of generality. More accurate simulations are not without problems of their own, as force fields created for common ionic liquids [32-38] are not straightforward to translate into ionic liquid crystal systems. The naïve perspective of adding an alkyl tail to ionic species is not always straightforward. For simulations of ionic liquids, specialized force fields must be developed [39] to capture both thermodynamic and dynamic properties accurately. Even so, different choices for the interactions lead to an enthalpy-entropy trade-off, which is seen in many coarse-grained force fields [40]. The precise interplay of van der Waals and Coulombic interactions, perhaps including polarizable interactions [41], in determining macroscopic properties can lead to very accurate predictions for one molecule, but a lack of transferability to other models. While some coarse-graining techniques, such as Effective Force Coarse-Graining [36], have proven to be transferable for certain series of ionic liquid crystals [37], an all-atom approach is preferred for greater interoperability between molecules. All-atom approaches have seen limited applications to ionic liquid crystal systems, though a few notable investigations exist. Importantly, these are limited in scope, examining structural features in a narrow temperature range or are focused primarily on shorter alkyl chains.

Several generic force-field parameter sets, including GAFF [42], UFF [43], and OPLS [44] have been parameterized for a large number of common and some less common chemical groups, allowing for novel materials to be simulated without parameterizing a new force field for each. As a standard model for all-atom force fields, a modified OPLS-AA force field [33,34] provides generality to many series of common ionic liquids, with various lengths of alkyl side chains. The applicability of this all-atom force field to long molecules remains to be thoroughly studied, hence examining the effects of all-atom force fields in these systems is of chief interest in our investigation here. This force field contains many classes of molecules, hence its transferability to various types of ionic liquid crystals would be useful in studying this burgeoning field of molecular systems engineering. 
We focus here on providing a detailed analysis of the phase behavior and dynamics using an all-atom model of 1-alkyl-3-methylimidazolium nitrate, compactly notated as $\left[\mathrm{C}_{n} \mathrm{MIm}\right]\left[\mathrm{NO}_{3}\right]$ (see Figure 1 for an example structure). With long alkyl tails, this ionic liquid system forms liquid crystalline phases $[21,45,46]$. Since the charge groups for both species are relatively localized, the charge-containing domains can be structured in specific ways that have an anisotropy in their conductivity [47]. The extra ordering, when compared to isotropic ionic liquids, allows for greater tuning of interactions in the design of molecular media for catalysis [48] or other applications. The phase behavior of this particular system has not been studied in the ionic liquid crystal limit (long alkyl chains) from an all-atom simulation standpoint. In what follows, we will characterize the ordering of this molecular system using atomistic simulations and analysis of the dynamics within.
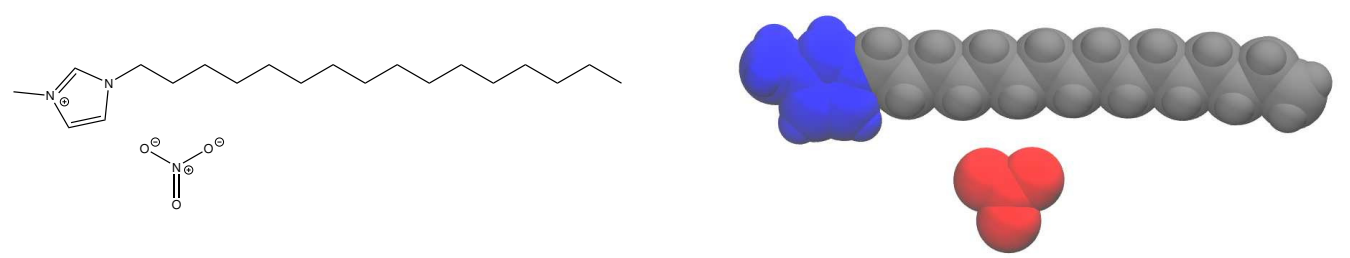

Figure 1. Chemical structure (left) and rendering of the atomistic model (right) of $\left[\mathrm{C}_{16} \mathrm{MIm}\right]\left[\mathrm{NO}_{3}\right]$. Colors in the rendered model signify the overall functional groups (blue: methyl imidazolium, gray: alkyl tail, red: nitrate).

\section{Methods}

A series of 12-, 14-, and 16-carbon chain length 1-alkyl-3-methylimidazolium $\left(\left[\mathrm{C}_{n} \mathrm{MIm}\right]^{+}\right)$and nitrate $\left(\left[\mathrm{NO}_{3}\right]^{-}\right)$ionic liquid crystals was simulated at constant atmospheric pressure (1 atm) in semi-isotropic or isotropic Parinello-Rahman simulation boxes with periodic boundary conditions. As discussed below, we performed temperature sweeps from $300 \mathrm{~K}$ to a maximum of $600 \mathrm{~K}$ using an ionic-liquid-modified OPLS-AA force field [33,34] in GROMACS 2016.3 [49], which contrasts with previous investigations using force fields such as CL\&AP [50] or GAFF [51].

The energy of these systems is calculated from several separate terms, based on harmonic bonds and angles, Fourier series-based torsions, and nonbonded interactions including Coulombic and Lennard-Jones terms. The functional form for the potential energy, $E$, of OPLS [44] is shown in Equation (1), in terms of the length of bond, $r$, angle between bonds, $\theta$, and torsional (or dihedral) angle, $\phi$.

$$
\begin{aligned}
E= & \sum_{i \in \text { bonds }} \frac{1}{2} k_{r, i}\left(r_{i}-r_{o, i}\right)^{2}+\sum_{i \in \text { angles }} \frac{1}{2} k_{\theta, i}\left(\theta_{i}-\theta_{o, i}\right)^{2}+ \\
& \sum_{i \in \text { torsions }} \frac{1}{2}\left[V_{1, i}\left(1+\cos \phi_{i}\right)+V_{2, i}\left(1-\cos 2 \phi_{i}\right)+V_{3, i}\left(1+\cos 3 \phi_{i}\right)+V_{4, i}\left(1-\cos 4 \phi_{i}\right)\right]+ \\
& \sum_{i \in \text { atoms } j>i} \frac{q_{i} q_{j} e^{2}}{r_{i j}}+4 \varepsilon_{i j}\left[\left(\frac{\sigma_{i j}}{r_{i j}}\right)^{12}-\left(\frac{\sigma_{i j}}{r_{i j}}\right)^{6}\right]
\end{aligned}
$$

The harmonic constants, $k_{r}$ and $k_{\theta}$, determine the strength of bonds and angles, respectively, around the minimum values of $r_{o}$ and $\theta_{o}$. The Fourier coefficients, $V_{n}$, define the torsional interaction strength within the molecules. The nonbonded interactions include the partial atomic charges, $q$, multiplied by the elementary charge, $e$, as well as the well depths, $\varepsilon$, and diameters, $\sigma$, of the Lennard-Jones potential for all particles.

To allow appropriate relaxation of the layered phases, semi-isotropic pressure coupling is used to allow fluctuations within the layers (in the $x$ - and $y$-directions) to decouple from the $z$-direction. 
To ensure proper representation of the fluid phase, an isotropic coupling is initiated after layers have melted. In these cases, the box dimensions from the previous temperature were used along with a constant pressure-constant temperature simulation to observe the isotropic liquid. Preliminary simulations using a fully charged model exhibited an overly stable crystal phase which was not observed to melt at temperatures less than $500 \mathrm{~K}$. Thus, in order to predict material properties, the charges were uniformly scaled by 0.8 , a modification commonly adopted in simulations of ionic liquids $[35,52]$. We found that this choice of scaling yielded behavior most consistent with prior studies $[45,53,54]$. However, the need to make such adjustments is indicative of inaccuracies in the force-field parameterization which necessitate eventual correction or reparameterization for ionic liquid crystalline systems. Importantly, we stress that while such a scaling may more accurately reproduce phase behavior and material dynamics, accurate resolution of charge transport and conductivity should retain $q=1.0$, and for these purposes the force balance should be corrected through other means [41].

To probe a range of temperatures, the systems were set up in a proposed crystal configuration (see Figure 2) and equilibrated for $20 \mathrm{~ns}$ at $300 \mathrm{~K}$, before increasing the temperature by discrete steps of $25 \mathrm{~K}$ up to $600 \mathrm{~K}$. The initial configuration is created by minimizing a single pair of ions and then copying and rotating in each of the Cartesian axes of the molecule, to acquire a unit cell of 8 ion pairs, with a point group of $D_{2 d}$. This is then copied and translationally arranged into 2 layers of $16 \times 16$ molecules, for a total of 512 ion pairs. This does not necessarily correspond to an equilibrated structure of the full system, particularly as multiple potential ionic arrangements are possible in crystalline states of ionic liquid crystal species [55], but merely provides a good starting point for investigations of layered structures. A "pre-conditioning" simulation of $5 \mathrm{~ns}$ at the starting temperature allows the hypothetical crystalline state (at $300 \mathrm{~K}$ ) to equilibrate the system partially before production simulations. The systems were allowed to equilibrate for $20 \mathrm{~ns}$, during the initial temperature ramp, before increasing to the next temperature, in series. Data from each temperature step were generated starting from the final configuration of the previous (lower) temperature, to allow for a more gradual thermal relaxation. Then, each temperature was allowed to equilibrate for an additional $280 \mathrm{~ns}$, in parallel, for a total simulation time of $300 \mathrm{~ns}$ at each temperature. This ramping scheme followed from a coarse-grained simulation of similar molecules [21] using discrete changes in temperature, followed by further equilibration.

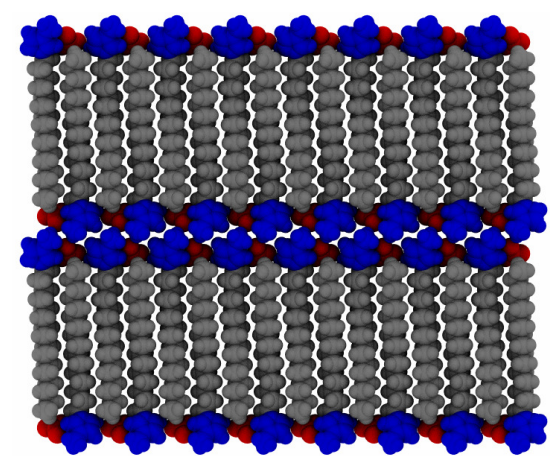

Figure 2. Initial configuration used in our simulation protocol. All of the alkyl tails are parallel, while neighboring molecules as a whole are antiparallel. Each dimension of each layer contains 16 evenly spaced molecules.

All simulations were run with a timestep of $1 \mathrm{fs}$, using a leap-frog integrator ("md" in GROMACS) and a Parrinello-Rahman barostat, with time constants of 2 ps and 1 ps, respectively. The compressibility was assumed to be that of water, or $4.5 \times 10^{-5}$ bar. These parameters are standard molecular dynamics values for fully atomistic simulations. As the initial configuration was set up to be somewhat unphysical, the box sizes quickly converged on more physical values. Since the system is 
symmetric around the $x$ - and $y$-directions, the aspect ratio of these dimensions was set to unity, while the $z$-direction's initial value was based on the length of the molecule when equilibrated in vacuum.

\section{Results}

As noted in the introduction, a typical material will undergo a phase change from a crystalline solid to an isotropic liquid, often observed through a sharp change in its volume. Liquid crystals, however, exhibit additional liquid phases of varying degrees of order. Upon heating our crystalline initial configurations, both ionic groups and aliphatic tails between the layers of charged groups can begin to move more freely among each other. Despite starting from a parallel initial configuration, these systems adopted a tilted, chevron-like ordering upon equilibration. This tilting is consistent with other simulations [51], but the alternating directions of tilt is an effect of our chosen initial configuration. The simplest way for the system to transform into a tilted configuration is by moving the central ionic layer in one direction as a whole, which induces the chevron-like ordering observed here. As the energetic effects holding each layer together are of different origin (van der Waals for the tails and Coulombic for the ionic groups), the different regions may have different effective melting temperatures, leading to further splitting of the observed phases. This is the case here, as depicted in Figure 3. At low temperatures, the layered structure of the crystal prevails, though thermal fluctuations cause some frustration within the crystal. In our system, this corresponds to disordering of the ionic placements, and some slight melting of the aliphatic tails (see Figure 3a), though there is still a strong degree of translational and orientational order, as will be discussed later. As this system is heated, the order among the tails begins to relax and the ionic domain becomes more disordered (see Figure $3 b$ ). Though we will show later this state exhibits structural and dynamic properties suggesting it is a distinct phase, a clear signature is not visible in the global thermodynamic variables we calculate, and hence we label this state also as a crystal. With additional heat, tail conformations melt significantly, though the layered structure is retained, forming a smectic-A phase [21]. Here, layers begin to pull slightly away from each other, and the spacing of layers is broadened as evidenced in Figure 3c. In molecules with a short alkyl chain, smectic phases are not observed, making such phases indicative of the class of ionic liquid crystals, though metastable phases which may include smectic-like order have also been reported [51]. The disorder between layers does not permeate through the layers, which means that the overall layered structure of the system does not differ between the crystalline and smectic phases. Nevertheless, there is a slight relaxation of the layer spacing in the smectic-A phase, since the alkyl chains are no longer optimally packed. Further heating of the system removes the layered structure (Figure 3d). While we refer to this as an isotropic liquid, there are clearly still some domains of charge segregation, indicative of the charged clusters often observed in simulations of ionic liquids [56,57].

The characterization of phase transitions may be done in multiple ways. For simulations of ionic liquid crystals, the intrinsic property of volume per ion pair (equivalent to an inverse number density) is often useful [21], as it can characterize significant jumps in the effective size of molecules, indicative of increased disorder. Within a single phase, volume as a function of temperature is close to linear, but a phase change is observed as a discontinuity or jump in this line. As Figure 4 shows, the transition between the crystalline and the smectic-A phase is seen quite easily for all three homologues. This is expected to have a significant signature, due to the change in layer spacing during this transition (see Figure 3c). The transition between the smectic-A phase and the isotropic liquid is less pronounced, but is confirmed through visualization or by characterizing the aliphatic ordering. These transitions are first order, and should be characterized by both a latent heat signature and a change in the heat capacity before and after the transition. Albeit, the phase transitions are less pronounced when examining this quantity, partially due to the fairly large temperature steps (25 K) utilized in our study (see Figure 5). 


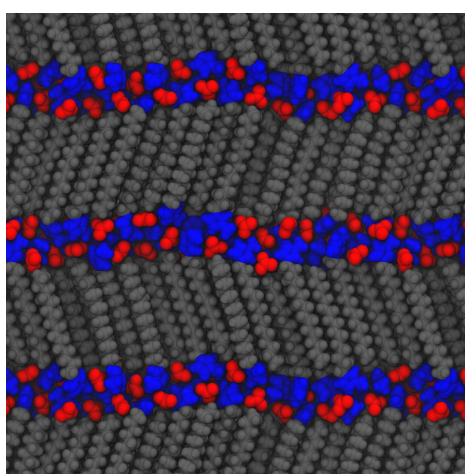

(a) Crystalline, $300 \mathrm{~K}$

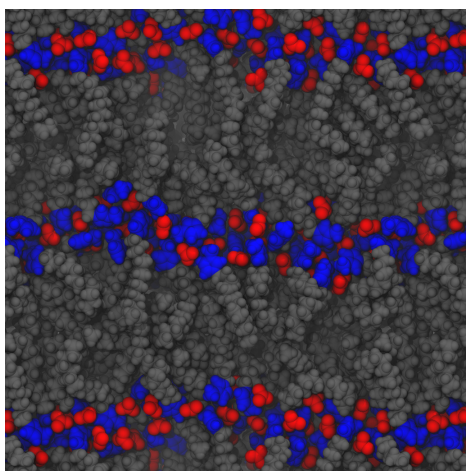

(c) Smectic-A, $500 \mathrm{~K}$

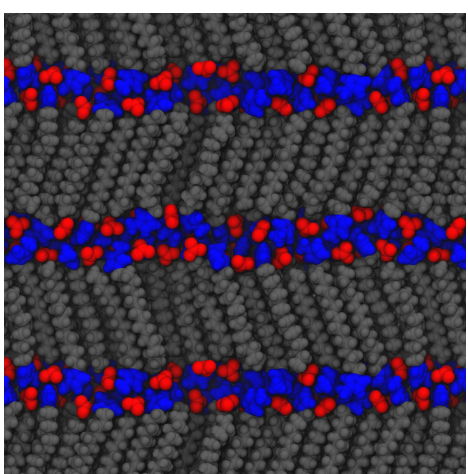

(b) Crystalline, $450 \mathrm{~K}$

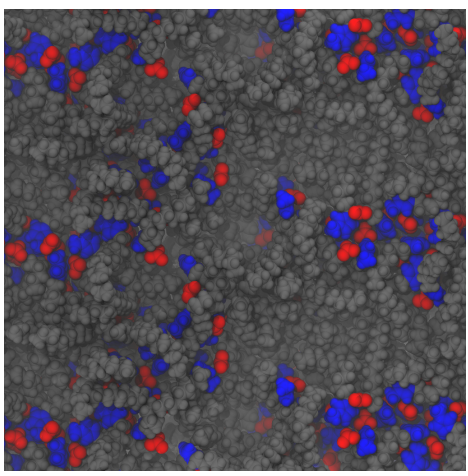

(d) Isotropic Liq., $600 \mathrm{~K}$

Figure 3. Observed phases in the ionic liquid crystal material, $\left[\mathrm{C}_{16} \mathrm{MIm}\right]\left[\mathrm{NO}_{3}\right]$. Typical configurations are presented from temperatures ranging between (a) $300 \mathrm{~K}$ and (d) $600 \mathrm{~K}$. The tilted layers in (a) are consistent with other models [51], though the alternating direction of tilt is most likely due to our simulations' proposed initial configurations. Frustrations within the crystal are visible in (a), as some aliphatic tails are bent within the layer and anions exhibit irregular placement. Intermediate temperatures illustrated in $(\mathbf{b}, \mathbf{c})$ depict the system on either side of the crystal-smectic phase transition. The isotropic liquid still exhibits segregation, but the layers have lost their long-range order, as evidenced by (d).

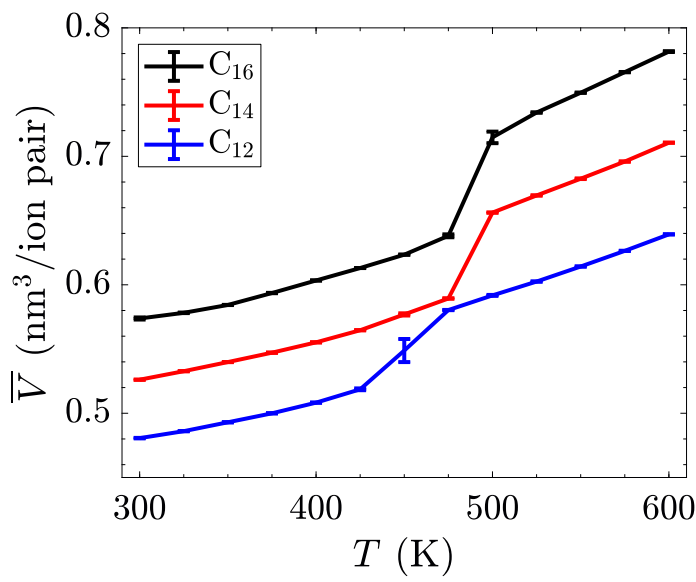

Figure 4. Intrinsic volume per ion pair as a function of temperature for three different alkyl chain lengths. The most dramatic jump in volume is between the crystalline and smectic phases. The transition between the smectic and isotropic phases is not apparent in this quantity. Transition temperatures are summarized in Table 1. 


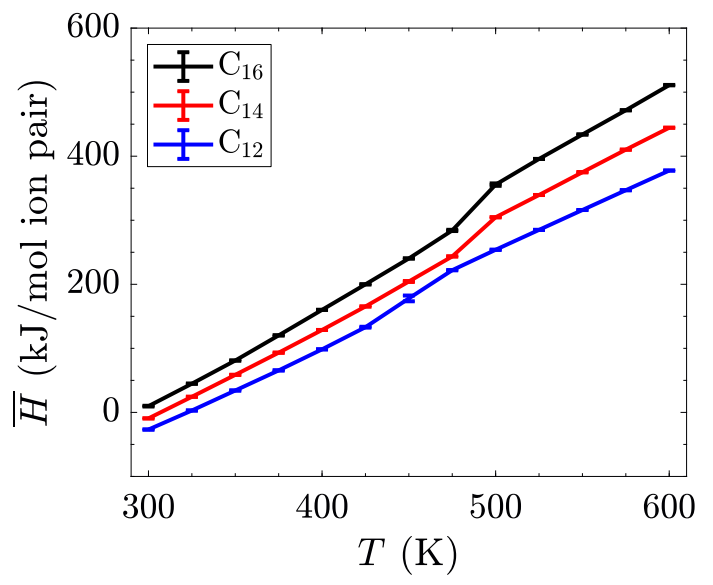

Figure 5. Intrinsic enthalpy as a function of temperature for three different alkyl chain lengths. The phase transitions are less pronounced in this system-wide property, but can be identified by the changes in slope of each line.

To better understand the ordering of liquid crystalline phases, we compute the orientational order parameter $S$ by defining a nematic director for each molecule from the axis with the smallest moment of inertia, $\hat{n}$. The $Q$-tensor [58] was subsequently calculated via Equation (2).

$$
\mathbf{Q}(t)=\frac{3}{2 N} \sum_{i=1}^{N}\left(\hat{\mathbf{n}}_{i}(t) \otimes \hat{\mathbf{n}}_{i}(t)-\frac{1}{3} \mathbf{I}\right)
$$

The orientational order parameter, $S$, is obtained from the largest positive eigenvalue of the characteristic equation of $\mathbf{Q}(t)$. The results, time-averaged for the last $200 \mathrm{~ns}$ of each temperature in the sweep, are summarized in Figure 6. By increasing the length of the alkyl side chain, the relative influence of the inter-layer Coulombic interactions and the van der Waals interactions stabilizes the smectic-A phases, thereby broadening the range of temperatures where the smectic-A is observed. Such behavior might be used to tune the position of the crystalline to smectic transition through the incorporations of softer ions or more structured tails with stronger van der Waals interactions.

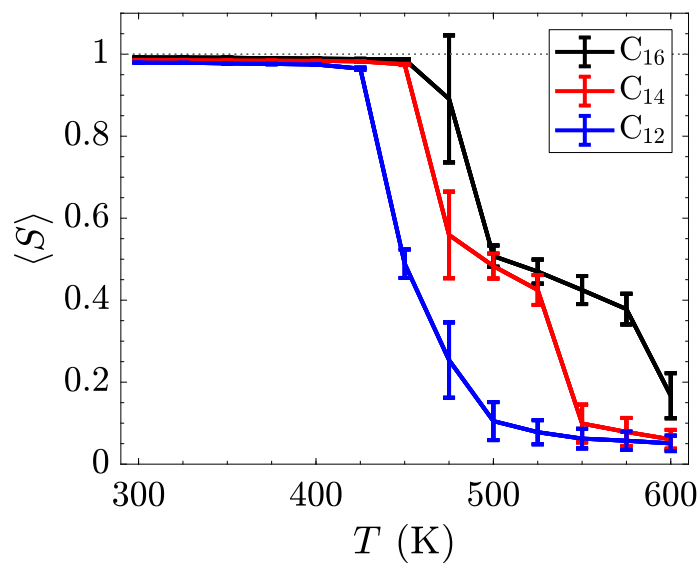

Figure 6. Nematic order parameter as a function of temperature for three different alkyl chain lengths, averaged over the last $200 \mathrm{~ns}$ of the trajectory. The layers are calculated separately and averaged, due to the opposite tilting directions in the crystal phase. Two distinct phase transitions can be observed as discontinuous jumps in this order parameter. The error bars are larger on temperatures near transition points, as the systems fluctuate more there, thus introducing more variance into trajectory-averaged properties. 
While the trends in the transition temperatures (listed in Table 1) of these atomistic simulations are similar to experimental measurements [45], the exact numbers differ between methods. These differences are also seen in simulations using transferable coarse-grained models [21]. This is indicative of a more permeating issue in molecular force fields; standard models are not able to capture the entire behavior of this class of materials. Often, the same procedure can yield accurate results for smaller homologues, but miss out on the behavior of longer aliphatic chains, as is observed [59] for long homologues of $\left[\mathrm{C}_{n} \mathrm{MIm}\right]\left[\mathrm{PF}_{6}\right]$.

In fact, when compared to experimental data [45], these transition temperatures are more comparable with $\left[\mathrm{C}_{n} \mathrm{MIm}\right][\mathrm{Cl}]$, which has a much smaller chloride anion, instead of the bulkier nitrate anion. The size of the chloride ion allows for a more focused effect of Coulombic interactions, as the charge is not spread over a large area. This, in turn, raises the temperature at which phase transitions occur, with more thermal energy required to overcome the Coulombic attraction between ions. This may not be the only effect causing the large gap separating computational and experimental transition temperature measurements. In particular, the polarizability inherent in the molecular system is poorly represented by the constant charges imposed within our model. This could potentially be addressed by adding polarizable sites [60] to allow for variation in the distribution of charge.

Table 1. Observed transition temperatures (upper bounds) between the various phases for this series of ionic liquid crystals. Guillet, et al. [45] did not observe any smectic phase for $\left[\mathrm{C}_{12} \mathrm{MIm}\right]\left[\mathrm{NO}_{3}\right]$, but observed the isotropic phase above $302 \mathrm{~K}$. As a function of alkyl tail length, the trends of transition temperatures are consistent with experimental results [45].

\begin{tabular}{ccccc}
\hline \multirow{2}{*}{ Species } & \multirow{2}{*}{ Transition } & \multicolumn{3}{c}{ Temperature, K } \\
\cline { 3 - 5 } & & This Work & Experimental [45] & Coarse-Grained [21] \\
\hline \multirow{2}{*}{$C_{12}$} & Crystal-Smectic-A & 450 & - & - \\
& Smectic-A-Isotropic & 500 & - & - \\
\hline \multirow{2}{*}{$C_{14}$} & Crystal-Smectic-A & 500 & 315 & - \\
& Smectic-A-Isotropic & 550 & 401 & 505 \\
& Crystal-Smectic-A & 500 & 320 & 560 \\
\hline \multirow{2}{*}{$C_{16}$} & Smectic-A-Isotropic & 575 & 457 & \\
\hline
\end{tabular}

While the data above were gathered from increasing temperature sweeps, we note that the smectic phase was also observed to spontaneously assemble from initially isotropic conditions above the melting transition. Simulations were performed at low temperatures starting from random configurations to ensure that our initial crystalline configuration was feasible for these systems. This confirmed that our system was equilibrated, as the phase was the same whether approached from lower or higher temperatures. If the layered structures were only metastable, these simulations would stay isotropic, instead of assembling into mesostructures spontaneously. It is possible that some hysteresis exists close to the melting temperatures due to metastability of the ordering behavior, as has been observed in recent simulations of ionic liquids and liquid crystals [51].

Another method of identifying phases is to monitor the mobility of molecules as the simulations progress. In the lower-temperature, crystalline regime, all atoms should have low mobility. As the material transitions to the smectic phase, the charge groups (the aromatic imidazolium rings and the anions) are fixed into thin layers, while the alkyl tails are free to move within their layer. The final phase transition sees mobility of all atoms of all species. Figure 7 shows the difference in mobility for select temperatures in these simulations. Near the transition temperature between crystalline and smectic phases, the long cations are still limited in their crystalline mobility, but the smaller nitrate ions are able to move much more freely, as observed as a divergence between the in-plane mobility of the nitrate anions and imidazolium cations in Figure $7 \mathrm{~b}$, which is still classified as the crystalline phase. This behavior near the transition temperature may signify an interesting design space, where the different ionic species have highly divergent mobilities. 

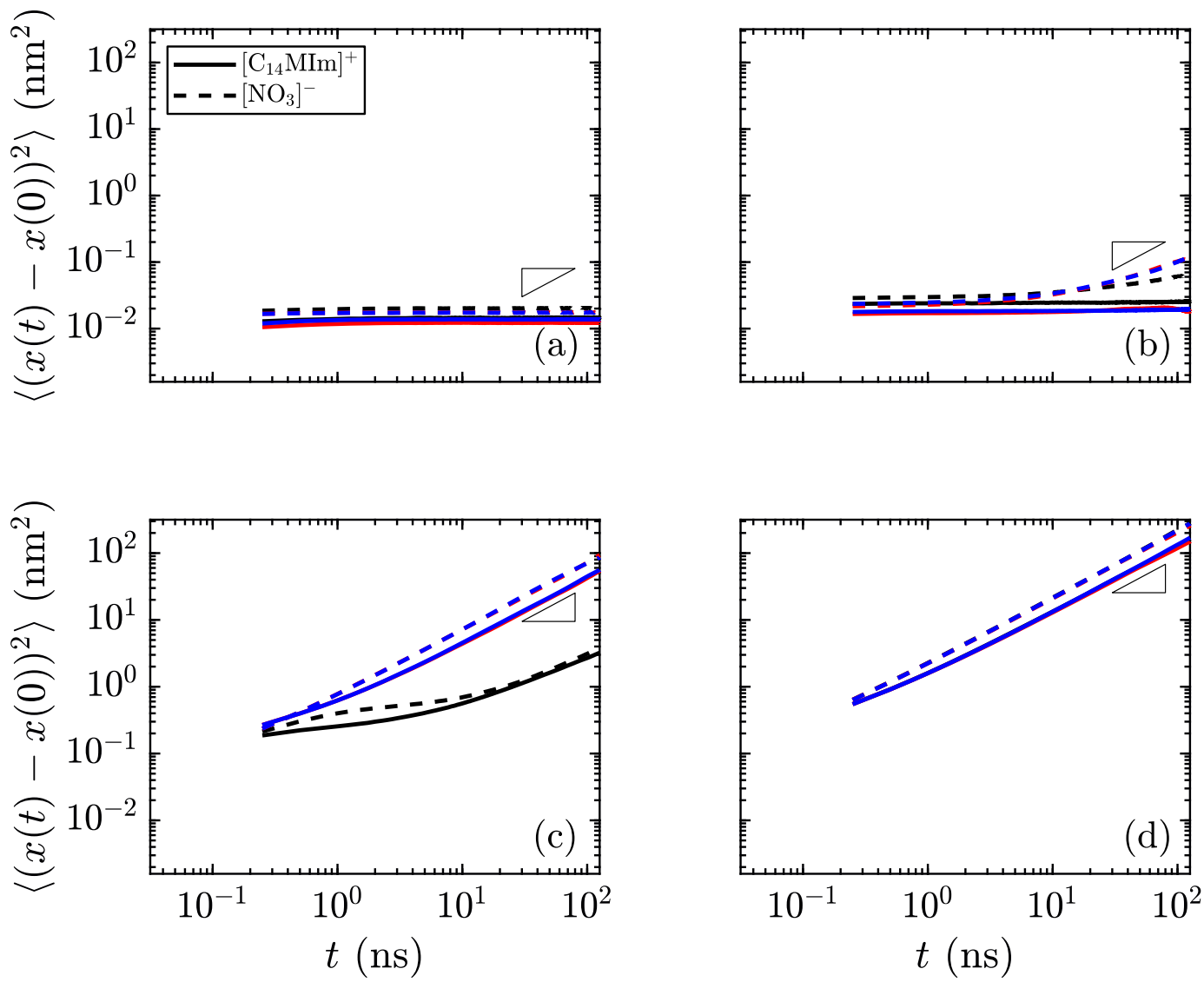

Figure 7. Mean-squared displacement of the species in $\left[\mathrm{C}_{14} \mathrm{MIm}\right]\left[\mathrm{NO}_{3}\right]$ as a function of time at temperatures of (a) $400 \mathrm{~K}$ in the crystalline state, (b) $450 \mathrm{~K}$ just before the transition to smectic, (c) $500 \mathrm{~K}$ in the smectic-A phase, and (d) $600 \mathrm{~K}$ isotropic liquid. Reference slopes of 1, indicated as small triangles, help to identify the linear diffusive regime. The colors signify the dimension (red: $x$, blue: $y$, black: $z$ ); the solid lines are for the long cations and the dotted lines are for the nitrate anions. The most distinctive difference is the divergence of the $z$-direction from the $x$ - and $y$-directions in the smectic-A phase, showing that transport within a layer is much simpler than through the layers. (Notes: (c) The $x$ - and $y$-directions are nearly overlapping. (d) All directions nearly collapse, indicating an isotropic phase.)

The different mobility signatures of the various phases allow for greater tunability in ion transport. For the phases unique to liquid crystals, the anisotropy of movement can control the transport of smaller species through the charged domains, as any small ions will remain confined within these regions rather than traversing between layers. By going to the low-temperature limit, all species have restricted mobility; the high-temperature limit allows for unhindered motion of all species. It is within these limits where the liquid crystalline properties are useful in determining the material order and directional conductivity for small charged species. These types of material show promise as having intrinsic methods for controlling ion (and hence charge) transport, which could prove useful in applications where the direction of ion transport is of prime importance. In particular, layered domains which form bulk smectic phases are likely to form conductive contacts with other layers at grain boundaries within a sample quenched into a solid or glass-like state, increasing the flow of charge carriers through the medium. Such topics are clear motivators for future work examining quantitatively the interplay of charged and apolar domains in these materials, and their influence on ion conduction between common cathode and anode materials.

The radial distribution function can also be indicative of structure in ionic liquid crystal phases. Due to the layered nature of these simulations, the three-dimensional form of $g(r)$ misses several properties of the low-temperature phases. Hence, the in-plane radial distribution function, $g_{\|}(r)$, is 
more relevant for this system, notwithstanding its collapsing of the z-direction. In an ideal crystal, this function will show sharp peaks at the locations of each molecule's nearest neighbors. However, introducing a nonzero temperature will spread out these peaks, as thermal fluctuations disrupt the perfect ordering. On the opposite side of the temperature range, an isotropic liquid will show no preference for order, therefore the averaged density around a certain molecule will be uniform. These profiles are shown in Figure 8, for the same temperatures as previous figures.
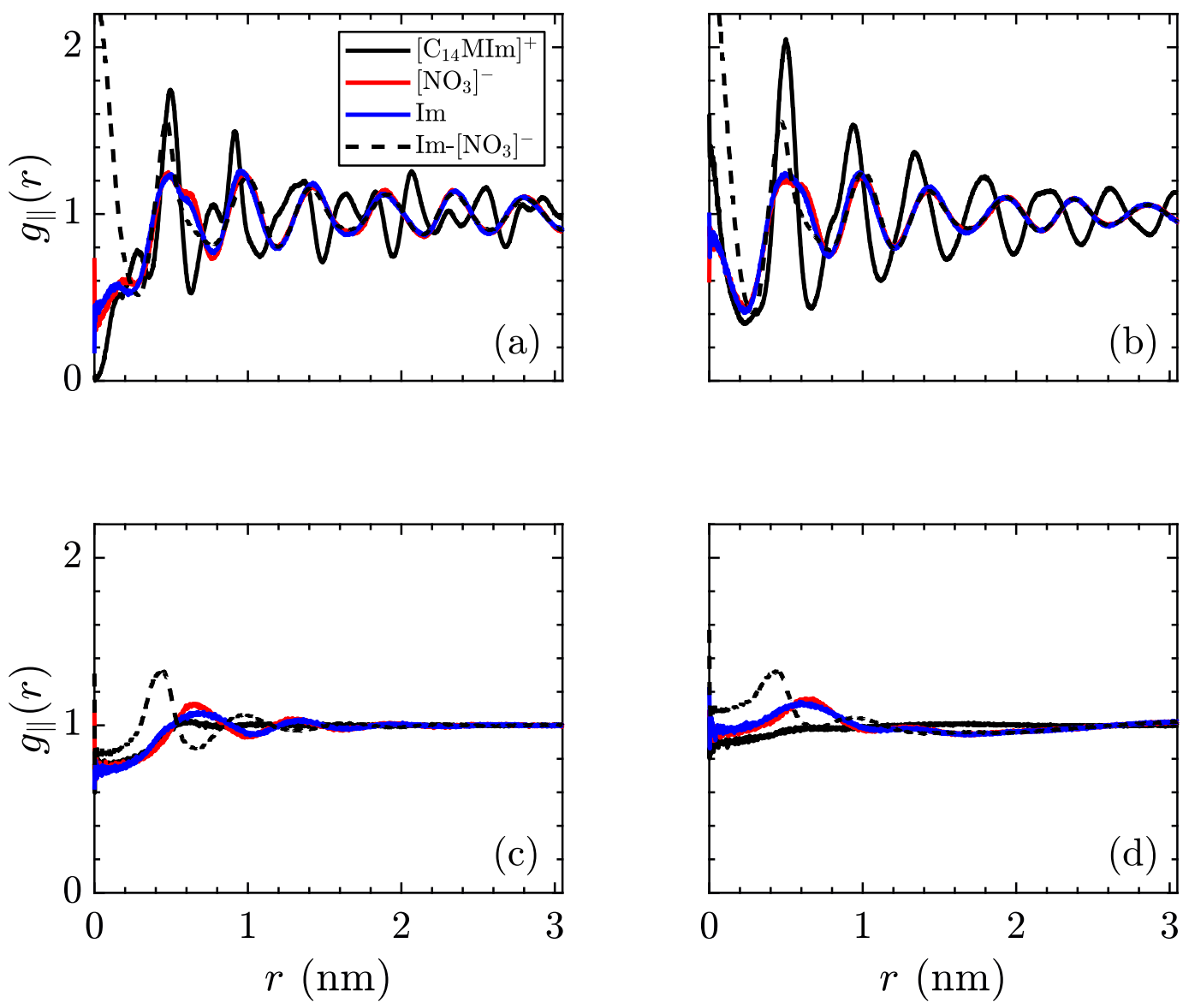

Figure 8. In-plane radial distribution function, $g_{\|}(r)$ of the species in $\left[\mathrm{C}_{14} \mathrm{MIm}\right]\left[\mathrm{NO}_{3}\right]$, trajectory-averaged over the last $200 \mathrm{~ns}$, at temperatures of (a) $400 \mathrm{~K}$ in the crystalline state, (b) $450 \mathrm{~K}$ just before the transition to the smectic-A state, (c) $500 \mathrm{~K}$ in the smectic-A phase, and (d) $600 \mathrm{~K}$ isotropic liquid. The solid lines signify distribution of a species relative to its own type (black: $\left[\mathrm{C}_{14} \mathrm{MIm}\right]^{+}$, red: $\left[\mathrm{NO}_{3}\right]^{-}$, blue: imidazolyl ring) while the dotted line denotes placement of $\left[\mathrm{NO}_{3}\right]^{-}$ions in relation to imidazolyl rings. From (a) to (b), a significant decrease in order of tail groups is observed, with the loss of secondary peaks, while the correlations also decay slightly more quickly as a function of distance. The first phase transition is characterized by the alkyl tails losing almost all order within the $x y$-plane. The phase transition to the isotropic liquid loses almost all structure, except for pairing of the opposite charges.

As with the mean-squared displacement, an interesting change occurs before the crystalline-smectic transition, where the charged moieties (both $\left[\mathrm{NO}_{3}\right]^{-}$and $\left[\mathrm{C}_{14} \mathrm{MIm}\right]^{+}$) begin to exhibit a more smoothed radial distribution function than the aliphatic tails. Coupled with the observations regarding ionic mobility, this is very suggestive of an intermediate phase appearing in advance of the smectic-A phase, where the ionic layers are melted, but the aliphatic tails retain crystal structure. As no signature is observed in our measurements of volume per ion pair and intrinsic enthalpy, there is insufficient evidence to state a phase transition exists, though more detailed 
measurements of the thermodynamic variables in this region should be able to detect if this is a transient condition or a separate equilibrium phase. After transitioning to the smectic-A phase, the centers of the charged groups continue to exhibit ordering, as the planes of ionic domains are still intact, but the alkyl tails are more disordered within the aliphatic domains. As previously mentioned, the high temperature smectic-isotropic transition only refers to melting of the layers and does not result in a fully isotropic liquid, as evidenced by the short-range structure of ionic groups, quickly decaying after a few nearest neighbors.

\section{Discussions \& Conclusions}

A fully atomistic investigation into the phase behavior of an imidazolium-based ionic liquid crystal series was performed. At these lengths of alkyl side chains, three distinct phases are observed for temperatures between $300 \mathrm{~K}$ and $600 \mathrm{~K}$ : crystalline, smectic-A, and an isotropic liquid. The length of the alkyl chain plays an important role in defining the conditions under which these molecular systems change phases. Using an all-atom model allows for greater transferability at a small computational cost, since many ionic liquid species were parameterized with the goal of interoperability between species.

While the general trends of the transition temperatures are correct, there are differences between this work and previous investigations [21]. The main source of disagreement is the type of model used: all-atom versus coarse-grained. Prior to this work, fully atomistic simulations of this degree have not been pursued. In addition, there may be added entropy due to the number of additional atoms to simulated. However, the trends for these long molecules extrapolate to suggest that $\left[\mathrm{C}_{10} \mathrm{MIm}\right]\left[\mathrm{NO}_{3}\right]$ does not have liquid crystal-like behavior, which is supported by previous investigations [45]. Importantly, we also observe an interesting dynamic behavior just prior to the crystalline-smectic transition which could be the hallmark of another phase or an intriguing metastable state; more detailed study is necessary to understand this state completely. While at even higher temperatures, there may be several non-layered mesophases that these systems exhibit; we do not observe any significant thermodynamic or structural changes aside from the crystalline-smectic and smectic-isotropic transitions for the temperatures studied here. Thus, due to the high temperatures needed to observe these phases, the characterization of these phases is likely of less practical interest.

Materials of this class may prove useful in various applications, such as forming a structured medium for batteries where the conductivity in the different directions can be tuned. At the specified temperatures, the charged moieties are confined to thin layers, with essentially no transfer between layers, which directs the mobility of ions. While the motions of this material were characterized here, the addition of other ions will require further investigations into diffusive transport through these materials.

Acknowledgments: M.J.Q. and J.K.W. greatly acknowledge computational resources provided by the Notre Dame Center for Research Computing (CRC), startup funds from the University of Notre Dame, and support from the Midwest Integrated Center for Computational Materials (MICCoM), a computational hub supported by the United States Department of Energy, Basic Energy Sciences division.

Author Contributions: M.J.Q. and J.K.W. designed the study, performed the research, analyzed the data and wrote the paper.

Conflicts of Interest: The authors declare no conflict of interest.

\section{Abbreviations}

The following abbreviations are used in this manuscript:

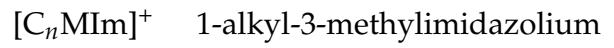

$\left[\mathrm{NO}_{3}\right]^{-} \quad$ nitrate

$\left[\mathrm{PF}_{6}\right]^{-} \quad$ hexafluorophosphate

$\left[\mathrm{BF}_{4}\right]^{-} \quad$ tetrafluoroborate

SDS sodium dodecyl sulfate 


\section{References}

1. Binnemans, K. Ionic Liquid Crystals. Chem. Rev. 2005, 105, 4148-4204. [CrossRef] [PubMed]

2. Mansueto, M.; Laschat, S. Ionic Liquid Crystals. In Handbook of Liquid Crystals; Wiley-VCH Verlag: Weinheim, Germany, 2014.

3. Wang, X.; Sternberg, M.; Kohler, F.T.; Melcher, B.U.; Wasserscheid, P.; Meyer, K. Long-alkyl-chain-derivatized imidazolium salts and ionic liquid crystals with tailor-made properties. RSC Adv. 2014, 4, 12476-12481. [CrossRef]

4. $\quad$ Lee, J.H.; Han, K.S.; Lee, J.S.; Lee, A.S.; Park, S.K.; Hong, S.Y.; Lee, J.C.; Mueller, K.T.; Hong, S.M.; Koo, C.M. Liquid Crystals: Facilitated Ion Transport in Smectic Ordered Ionic Liquid Crystals (Adv. Mater. 42/2016). Adv. Mater. 2016, 28, 9439. [CrossRef]

5. Axenov, K.V.; Laschat, S. Thermotropic ionic liquid crystals. Materials 2011, 4, 206-259. [CrossRef]

6. Goossens, K.; Lava, K.; Bielawski, C.W.; Binnemans, K. Ionic liquid crystals: Versatile materials. Chem. Rev. 2016, 116, 4643-4807. [CrossRef]

7. Gordon, C.M.; Holbrey, J.D.; Kennedy, A.R.; Seddon, K.R. Ionic liquid crystals: Hexafluorophosphate salts. J. Mater. Chem. 1998, 8, 2627-2636. [CrossRef]

8. Kouwer, P.H.; Swager, T.M. Synthesis and mesomorphic properties of rigid-core ionic liquid crystals. J. Am. Chem. Soc. 2007, 129, 14042-14052. [CrossRef]

9. Chandrasekhar, S. Liquid Crystals; Cambridge University Press: New York, NY, USA, 1993.

10. De Gennes, P.G.; Prost, J. The Physics of Liquid Crystals (International Series of Monographs on Physics); Clarendon Press: Oxford, UK, 1995.

11. Toledo Hijo, A.A.; Maximo, G.J.; Costa, M.C.; Cunha, R.L.; Pereira, J.F.; Kurnia, K.A.; Batista, E.A.; Meirelles, A.J. Phase Behavior and Physical Properties of New Biobased Ionic Liquid Crystals. J. Phys. Chem. B 2017, 121, 3177-3189. [CrossRef]

12. Lindblom, G.; Larsson, K.; Johansson, L.; Fontell, K.; Forsén, S. The cubic phase of monoglyceride-water systems. Arguments for a structure based upon lamellar bilayer units. J. Am. Chem. Soc. 1979, 101, 5465-5470. [CrossRef]

13. Lester, C.L.; Smith, S.M.; Colson, C.D.; Guymon, C.A. Physical properties of hydrogels synthesized from lyotropic liquid crystalline templates. Chem. Mater. 2003, 15, 3376-3384. [CrossRef]

14. Tang, K.; Green, M.M.; Cheon, K.S.; Selinger, J.V.; Garetz, B.A. Chiral conflict. The effect of temperature on the helical sense of a polymer controlled by the competition between structurally different enantiomers: From dilute solution to the lyotropic liquid crystal state. J. Am. Chem. Soc. 2003, 125, 7313-7323. [CrossRef] [PubMed]

15. Kékicheff, P.; Grabielle-Madelmont, C.; Ollivon, M. Phase diagram of sodium dodecyl sulfate-water system: 1. A calorimetric study. J. Colloid Interface Sci. 1989, 131, 112-132. [CrossRef]

16. Freemantle, M. An Introduction to Ionic Liquids; Royal Society of Chemistry: Cambridge, UK, 2010.

17. Ubbelohde, A.R.; Michels, H.J.; Duruz, J.J. Liquid Crystals in Molten Salt Systems. Nature 1970, 228, 50-52. [CrossRef]

18. Ramírez-González, P.E.; Ren, G.; Saielli, G.; Wang, Y. Effect of Ion Rigidity on Physical Properties of Ionic Liquids Studied by Molecular Dynamics Simulation. J. Phys. Chem. B 2016, 120, 5678-5690. [CrossRef]

19. Lee, C.K.; Huang, H.W.; Lin, I.J.B. Simple amphiphilic liquid crystalline 1-alkylimidazolium salts. A new solvent system providing a partially ordered environment. Chem. Commun. 2000, 1911-1912. [CrossRef]

20. Ji, Y.; Shi, R.; Wang, Y.; Saielli, G. Effect of the Chain Length on the Structure of Ionic Liquids: From Spatial Heterogeneity to Ionic Liquid Crystals. J. Phys. Chem. B 2013, 117, 1104-1109. [CrossRef]

21. Saielli, G. MD simulation of the mesomorphic behaviour of 1-hexadecyl-3-methylimidazolium nitrate: Assessment of the performance of a coarse-grained force field. Soft Matter 2012, 8, 10279-10287. [CrossRef]

22. Welton, T. Room-temperature ionic liquids. Solvents for synthesis and catalysis. Chem. Rev. 1999, 99, 2071-2084. [CrossRef]

23. Rogers, R.D.; Seddon, K.R. Ionic liquids-solvents of the future? Science 2003, 302, 792-793. [CrossRef]

24. Matsumoto, H.; Sakaebe, H.; Tatsumi, K. Preparation of room temperature ionic liquids based on aliphatic onium cations and asymmetric amide anions and their electrochemical properties as a lithium battery electrolyte. J. Power Source 2005, 146, 45-50. [CrossRef] 
25. Galiński, M.; Lewandowski, A.; Stępniak, I. Ionic liquids as electrolytes. Electrochim. Acta 2006, 51, 5567-5580. [CrossRef]

26. Bates, E.D.; Mayton, R.D.; Ntai, I.; Davis, J.H. $\mathrm{CO}_{2}$ capture by a task-specific ionic liquid. J. Am. Chem. Soc. 2002, 124, 926-927. [CrossRef] [PubMed]

27. Figueroa, J.D.; Fout, T.; Plasynski, S.; Mcllvried, H.; Srivastava, R.D. Advances in $\mathrm{CO}_{2}$ capture technology-The US Department of Energy's Carbon Sequestration Program. Int. J. Greenh. Gas Control 2008, 2, 9-20. [CrossRef]

28. Klimusheva, G.; Mirnaya, T.; Garbovskiy, Y. Versatile nonlinear-optical materials based on mesomorphic metal alkanoates: Design, properties, and applications. Liquid Cryst. Rev. 2015, 3, 28-57. [CrossRef]

29. Kitazawa, Y.; Ueno, K.; Watanabe, M. Advanced Materials Based on Polymers and Ionic Liquids. Chem. Rec. 2017, 17, 1-20. [CrossRef]

30. Gay, J.; Berne, B. Modification of the overlap potential to mimic a linear site-site potential. J. Chem. Phys. 1981, 74, 3316-3319. [CrossRef]

31. Saielli, G.; Margola, T.; Satoh, K. Tuning Coulombic interactions to stabilize nematic and smectic ionic liquid crystal phases in mixtures of charged soft ellipsoids and spheres. Soft Matter 2017, 14, 5204-5213. [CrossRef]

32. Lopes, J.N.C.; Deschamps, J.; Pádua, A.A. Modeling ionic liquids using a systematic all-atom force field. J. Phys. Chem. B 2004, 108, 2038-2047. [CrossRef]

33. Sambasivarao, S.V.; Acevedo, O. Development of OPLS-AA Force Field Parameters for 68 Unique Ionic Liquids. J. Chem. Theory Comput. 2009, 5, 1038-1050. [CrossRef]

34. Doherty, B.; Zhong, X.; Gathiaka, S.; Li, B.; Acevedo, O. Revisiting OPLS force field parameters for ionic liquid simulations. J. Chem. Theory Comput. 2017, 13, 6131-6145. [CrossRef]

35. Bhargava, B.L.; Balasubramanian, S. Refined potential model for atomistic simulations of ionic liquid [bmim][PF6]. J. Chem. Phys. 2007, 127, 114510. [CrossRef] [PubMed]

36. Wang, Y.; Noid, W.G.; Liu, P.; Voth, G.A. Effective force coarse-graining. Phys. Chem. Chem. Phys. 2009, 11, 2002-2015. [CrossRef] [PubMed]

37. Wang, Y.; Feng, S.; Voth, G.A. Transferable coarse-grained models for ionic liquids. J. Chem. Theory Comput. 2009, 5, 1091-1098. [CrossRef] [PubMed]

38. Dommert, F.; Wendler, K.; Berger, R.; Delle Site, L.; Holm, C. Force fields for studying the structure and dynamics of ionic liquids: A critical review of recent developments. ChemPhysChem 2012, 13, 1625-1637. [CrossRef] [PubMed]

39. Morrow, T.I.; Maginn, E.J. Molecular dynamics study of the ionic liquid 1-n-butyl-3-methylimidazolium hexafluorophosphate. J. Phys. Chem. B 2002, 106, 12807-12813. [CrossRef]

40. Chaimovich, A.; Shell, M.S. Relative entropy as a universal metric for multiscale errors. Phys. Rev. E 2010, 81, 060104. [CrossRef] [PubMed]

41. Schröder, C. Comparing reduced partial charge models with polarizable simulations of ionic liquids. Phys. Chem. Chem. Phys. 2012, 14, 3089-3102. [CrossRef]

42. Wang, J.; Wolf, R.M.; Caldwell, J.W.; Kollman, P.A.; Case, D.A. Development and testing of a general amber force field. J. Comput. Chem. 2004, 25, 1157-1174. [CrossRef]

43. Rappé, A.K.; Casewit, C.J.; Colwell, K.; Goddard, W., III; Skiff, W. UFF, a full periodic table force field for molecular mechanics and molecular dynamics simulations. J. Am. Chem. Soc. 1992, 114, 10024-10035. [CrossRef]

44. Jorgensen, W.L.; Maxwell, D.S.; Tirado-Rives, J. Development and testing of the OPLS all-atom force field on conformational energetics and properties of organic liquids. J. Am. Chem. Soc. 1996, 118, 11225-11236. [CrossRef]

45. Guillet, E.; Imbert, D.; Scopelliti, R.; Bünzli, J.C.G. Tuning the emission color of europium-containing ionic liquid-crystalline phases. Chem. Mater. 2004, 16, 4063-4070. [CrossRef]

46. El Seoud, O.A.; Pires, P.A.R.; Abdel-Moghny, T.; Bastos, E.L. Synthesis and micellar properties of surface-active ionic liquids: 1-alkyl-3-methylimidazolium chlorides. J. Colloid Interface Sci. 2007, 313, $296-304$. [CrossRef] [PubMed]

47. Kato, T. Self-assembly of phase-segregated liquid crystal structures. Science 2002, 295, 2414-2418. [CrossRef] [PubMed] 
48. Mehnert, C.P.; Cook, R.A.; Dispenziere, N.C.; Afeworki, M. Supported ionic liquid catalysis-A new concept for homogeneous hydroformylation catalysis. J. Am. Chem. Soc. 2002, 124, 12932-12933. [CrossRef] [PubMed]

49. Berendsen, H.J.; van der Spoel, D.; van Drunen, R. GROMACS: A message-passing parallel molecular dynamics implementation. Comput. Phys. Commun. 1995, 91, 43-56. [CrossRef]

50. Saielli, G. Fully atomistic simulations of the ionic liquid crystal $\left[\mathrm{C}_{16} \mathrm{MIM}\right]\left[\mathrm{NO}_{3}\right]$ : Orientational order parameters and voids distribution. J. Phys. Chem. B 2016, 120, 2569-2577. [CrossRef]

51. Cao, W.; Wang, Y.; Saielli, G. Metastable State during Melting and Solid-Solid Phase Transition of $\left[\mathrm{C}_{n} \mathrm{Mim}\right]\left[\mathrm{NO}_{3}\right](n=4-12)$ Ionic Liquids by Molecular Dynamics Simulation. J. Phys. Chem. B 2018, 122, 229-239. [CrossRef]

52. Zhang, Y.; Maginn, E.J. A simple AIMD approach to derive atomic charges for condensed phase simulation of ionic liquids. J. Phys. Chem. B 2012, 116, 10036-10048. [CrossRef]

53. Vanyúr, R.; Biczók, L.; Miskolczy, Z. Micelle formation of 1-alkyl-3-methylimidazolium bromide ionic liquids in aqueous solution. Colloids Surf. A Physicochem. Eng. Asp. 2007, 299, 256-261. [CrossRef]

54. Strechan, A.; Kabo, A.; Paulechka, Y.; Blokhin, A.; Kabo, G.; Shaplov, A.; Lozinskaya, E. Thermochemical properties of 1-butyl-3-methylimidazolium nitrate. Thermochim. Acta 2008, 474, 25-31. [CrossRef]

55. Alvarez Fernandez, A.; Kouwer, P.H. Key Developments in Ionic Liquid Crystals. Int. J. Mol. Sci. 2016, 17, 731. [CrossRef] [PubMed]

56. Triolo, A.; Russina, O.; Bleif, H.J.; Di Cola, E. Nanoscale segregation in room temperature ionic liquids. J. Phys. Chem. B 2007, 111, 4641-4644. [CrossRef] [PubMed]

57. Shimizu, K.; Gomes, M.F.C.; Pádua, A.A.; Rebelo, L.P.; Lopes, J.N.C. Three commentaries on the nano-segregated structure of ionic liquids. J. Mol. Struct. THEOCHEM 2010, 946, 70-76. [CrossRef]

58. Mottram, N.J.; Newton, C.J. Introduction to Q-tensor theory. arXiv 2014, arXiv:1409.3542.

59. Zhang, Y.; Maginn, E.J. Molecular dynamics study of the effect of alkyl chain length on melting points of $\left[\mathrm{C}_{n} \mathrm{MIM}\right]\left[\mathrm{PF}_{6}\right]$ ionic liquids. Phys. Chem. Chem. Phys. 2014, 16, 13489-13499. [CrossRef]

60. Halgren, T.A.; Damm, W. Polarizable force fields. Curr. Opin. Struct. Biol. 2001, 11, 236-242. [CrossRef]

(C) 2018 by the authors. Licensee MDPI, Basel, Switzerland. This article is an open access article distributed under the terms and conditions of the Creative Commons Attribution (CC BY) license (http:/ / creativecommons.org/licenses/by/4.0/). 